\title{
Racial Disparities in Bariatric Surgery Complications and Mortality Using the MBSAQIP Data Registry
}

\author{
Leonard K. Welsh ${ }^{1} \cdot$ Andrew R. Luhrs ${ }^{1} \cdot$ Gerardo Davalos $^{1} \cdot$ Ramon Diaz $^{1} \cdot$ Andres Narvaez $^{1}$. Juan Esteban Perez ${ }^{1}$. \\ Reginald Lerebours $^{2} \cdot$ Maragatha Kuchibhatla $^{2}$. Dana D. Portenier ${ }^{1} \cdot$ Alfredo D. Guerron $^{1}$ (D)
}

Published online: 9 May 2020

(C) Springer Science+Business Media, LLC, part of Springer Nature 2020

\begin{abstract}
Background Racial disparities in postoperative complications have been demonstrated in bariatric surgery, yet the relationship of race to complication severity is unknown.

Study Design Adult laparoscopic primary bariatric procedures were queried from the 2015 and 2016 MBSAQIP registry. Adjusted logistic and multinomial regressions were used to examine the relationships between race and 30-day complications categorized by the Clavien-Dindo grading system.

Results A total of 212,970 patients were included in the regression analyses. For Black patients, readmissions were higher $(\mathrm{OR}=$ $1.39, p<0.0001)$ and the odds of a Grade $1,3,4$, or 5 complication were increased compared with White patients $(\mathrm{OR}=1.21$, $p<0.0001 ; \mathrm{OR}=1.21, p<0.0001 ; \mathrm{OR}=1.22, p=0.01$; and $\mathrm{OR}=1.43, p=0.04)$ respectively. The odds of a Grade 3 complication for Hispanic patients were higher compared with White patients $(\mathrm{OR}=1.59, p<0.0001)$.

Conclusion Black patients have higher odds of readmission and multiple grades of complications (including death) compared with White patients. Hispanic patients have higher odds of a Grade 3 complication compared with White patients. No significant differences were found with other races. Specific causes of these disparities are beyond the limitations of the dataset and stand as a topic for future inquiry.
\end{abstract}

Keywords MBSAQIP $\cdot$ Racial disparities $\cdot$ Outcomes $\cdot$ Clavien-Dindo

\section{Introduction}

Obesity is the most prevalent chronic disease and a leading cause of morbidity and mortality in the USA. More than one-third of the US adult population meets the criteria for a diagnosis of obesity [1]. Obesity does not affect all racial and ethnic groups equally. According to the National Center for Health Statistics, non-Hispanic African American women are the most at-risk group, followed by Hispanic, non-Hispanic whites, and Asian women [1]. Similar trends are found among men. Weight loss surgery

Alfredo D. Guerron

alfredo.guerron-cruz@duke.edu

1 Division of Metabolic and Weight Loss Surgery, Department of Surgery, Duke University, 407 Crutchfield St., Durham, NC 27704, USA

2 Department of Biostatistics and Bioinformatics, Duke University, 2424 Erwin Rd, Durham 27710, USA remains the most effective treatment for moderate to severe obesity and resolution of obesity-related comorbidities [2-7]. Most bariatric operations have very low rates of morbidity and mortality [8] and are among the more commonly performed operations in the USA [9].

Racial disparities in operative outcomes have been demonstrated in many major surgical operations [10-12] and parallel findings have been demonstrated in bariatric surgery [13-16]. There is a general consensus that racial and ethnic minorities lose less weight than White patients after bariatric surgery due to a host of influential factors such as the selection of surgery type, sex, age, and access to care $[15,17,18]$. The significant metabolic benefits after bariatric surgery are welldemonstrated; however, minority populations respond with less weight loss and less durable comorbidity resolution [19, 20]. In a retrospective study, Istfan et al. [21] found that Black patients had a higher rate of hemoglobin Alc level increase 2 years after Roux-en-Y gastric bypass, while these levels remained stable in Hispanic and White patients. To further complicate the issue, Wood et al. [15] found that despite less 
weight loss 1 year after surgery, remission of sleep apnea and gastroesophageal reflux disease was higher in Black patients.

Further disparities in morbidity and mortality after bariatric surgery have been observed $[15,22,23]$. Black patients undergoing bariatric surgery demonstrate higher in-hospital and 30-day mortality and increased rates of adverse events after bariatric surgery compared with counterparts of other races $[14,16,24]$. Black patients also have worse short-term outcomes, including longer hospital length of stay, increased readmissions, and higher rates of 30-day complications [14, 15]. However, the severity of post-bariatric surgery complications and the relationship to patient race remain unclear.

Previous studies have been limited to institutional or statespecific data and only a few studies have used large national databases. The objective of this study is to identify disparities in reported complications using 30-day data from the Metabolic and Bariatric Surgery Accreditation and Quality Improvement Program (MBSAQIP) Participant Use Data File. The reported complications contained in the data set can be further categorized by severity using the ClavienDindo grading system [25]. A better understanding of the complication grades can assist in patient selection, perioperative counseling, and improvement of patient care.

\section{Methods}

The MBSAQIP data registry consists of annual data from metabolic and bariatric operations performed at centers accredited by the American College of Surgeons and the American Society for Metabolic and Bariatric Surgery. The MBSAQIP represents the largest bariatric-specific clinical dataset and assists in providing high-quality care for bariatric surgical patients by following a rigorous review process and maintaining certain standards of practice. Data are abstracted from medical records by certified reviewers and all accredited centers report their outcomes to the MBSAQIP database devoid of identifying data. This database serves as a valuable resource for clinical research and acts as the ideal source to investigate racial outcome disparities on a national scale.

Duke University institutional review board determined analysis of the MBSAQIP database is exempt from review under the category of previously collected anonymous data. The 2015 and 2016 MBSAQIP Participant Use Files were queried for subjects that underwent a laparoscopic primary bariatric operation of Roux-en-Y gastric bypass, sleeve gastrectomy, or duodenal switch using the appropriate CPT code. Subjects that had a revision/conversion principal operative procedure were under the age of 18, underweight (with a BMI of less than 18.5) post-operation, or were of an unknown race were then excluded from the dataset. Subjects that did not have complete data for the relevant covariates of interest were also excluded. The primary outcomes were 30 -day readmission and the Clavien-Dindo complication grade that corresponds to that subject's most severe complication. Clavien-Dindo grade definitions and associated MBSAQIP reported variables are described in Table 1 [14, 16, 26-37].

Summary data regarding demographics, comorbidity, and outcome information were collected. Categorical variables are summarized with frequency counts and percentages; continuous variables are summarized with means with standard deviations and ranges. The original "RACE" and "HISPANIC" ethnicity variables were used to create a new "Race/ Ethnicity" variable. Subjects were classified as "Hispanic" (regardless of race) if [HISPANIC $=$ "Yes"]. The remaining non-Hispanic subjects were classified according to the "RACE" variable, with "American Indian or Alaska Native" and "Native Hawaiian or Other Pacific Islander" subjects grouped into one "Other" category. Non-Hispanic subjects with [RACE = "Unknown/Not Reported"] were classified as missing. Differences between different racial groups for selected variables were assessed using Kruskal-Wallis tests for continuous variables, and Chi-Square tests for categorical variables. A multivariable, logistic regression analysis was conducted to model the relationship between race and 30-day readmission, after adjusting for relevant variables as covariates (Table 2). A multivariable, multinomial logistic regression model was also postulated to model the relationship between race and respective Clavien-Dindo grade (compared with having no complications) after adjusting for relevant variables as covariates. An ordinal logistic regression model was not used to assess this second relationship because the proportional odds assumption was not met. The covariates that were included in both models are summarized in Table 3, along with the procedure, postoperative BMI, and readmission information. Statistical analyses were conducted using SAS 9.4 (SAS Institute Inc., Cary, NC).

\section{Results}

Initial query of the 2015 and 2016 MBSAQIP databases generate 411,972 unique participates. After implementing primary operation and operation type inclusion criteria, 329,681 participants remained. After applying the exclusion criteria (revision/conversion principal operative procedure, underweight with a BMI of 18.5 , age under 18 , or were of an unknown race), there were 288,844 participants remaining in the dataset. After removing the participants that did not have complete data for the variables included in the regression models, 212,970 subjects remained. There were 75,874 participants with missing data $(26.3 \%$ of the 288,844 participants) (Fig. 1). Of the 212,970 subjects with complete data included in the analysis, 44,478 (20.9\%) subjects were male. The average age of subjects in the cohort was 44.6 (SD 12.0) years. A total of 145,007 (68.1\%) subjects were non- 
Table 1 Clavien-Dindo classification of surgical complications

\begin{tabular}{|c|c|c|}
\hline Grade & Definition & MBSAQIP variables \\
\hline \multirow[t]{4}{*}{ I } & \multirow{3}{*}{$\begin{array}{l}\text { Any deviation from the normal postoperative course without the need for } \\
\text { pharmacological treatment or surgical, endoscopic, and radiological } \\
\text { interventions }\end{array}$} & $\begin{array}{l}\text { Superficial incisional } \\
\quad \text { SSI }\end{array}$ \\
\hline & & $\begin{array}{c}\text { Dehydration } \\
\text { treatment }\end{array}$ \\
\hline & & $\begin{array}{l}\text { Emergency } \\
\text { department visit }\end{array}$ \\
\hline & $\begin{array}{l}\text { Allowed therapeutic regimens are the following: drugs as antiemetics, } \\
\text { antipyretics, analgesics, diuretics, electrolytes, and physiotherapy. This } \\
\text { grade also includes wound infections opened at the bedside }\end{array}$ & $\begin{array}{l}\text { Peripheral nerve } \\
\text { injury }\end{array}$ \\
\hline \multirow[t]{6}{*}{ II } & \multirow{4}{*}{$\begin{array}{l}\text { Requiring pharmacological treatment with drugs or other than such allowed } \\
\text { for grade I complications }\end{array}$} & Pneumonia \\
\hline & & Blood transfusion \\
\hline & & $\begin{array}{l}\text { Urinary tract } \\
\text { infection }\end{array}$ \\
\hline & & $\begin{array}{l}\text { VTE requiring } \\
\text { treatment }\end{array}$ \\
\hline & \multirow[t]{2}{*}{$\begin{array}{l}\text { Blood transfusions and transfusions and total parenteral nutrition are also } \\
\text { included }\end{array}$} & $\begin{array}{l}\text { Anticoagulation for } \\
\text { VTE/PE }\end{array}$ \\
\hline & & Clostridium difficile \\
\hline \multirow[t]{7}{*}{ III } & \multirow[t]{7}{*}{ Requiring surgical, endoscopic, or radiological intervention } & Wound disruption \\
\hline & & Incisional hernia \\
\hline & & Deep incisional SSI \\
\hline & & Organ space SSI \\
\hline & & $\begin{array}{l}\text { Drain placement } \\
\text { after } 30 \text { days }\end{array}$ \\
\hline & & $\begin{array}{l}\text { Reoperation within } \\
30 \text { days }\end{array}$ \\
\hline & & $\begin{array}{l}\text { Intervention within } \\
30 \text { days }\end{array}$ \\
\hline \multirow[t]{11}{*}{ IV } & \multirow{11}{*}{$\begin{array}{l}\text { Life-threatening complication (including CNS complications*) requiring } \\
\text { IC/ICU management }\end{array}$} & Acute renal failure \\
\hline & & $\begin{array}{l}\text { Progressive renal } \\
\text { insufficiency }\end{array}$ \\
\hline & & $\begin{array}{l}\text { Myocardial } \\
\text { infarction }\end{array}$ \\
\hline & & Stroke/CVA \\
\hline & & Ventilator $>48 \mathrm{~h}$ \\
\hline & & $\begin{array}{l}\text { Unplanned } \\
\text { intubation }\end{array}$ \\
\hline & & $\begin{array}{l}\text { Pulmonary } \\
\text { embolism }\end{array}$ \\
\hline & & Cardiac arrest \\
\hline & & Coma $>24 \mathrm{~h}$ \\
\hline & & Sepsis \\
\hline & & Septic shock \\
\hline V & Death of a patient & 30-day mortality \\
\hline
\end{tabular}

*Brain hemorrhage, ischemic stroke, and subarachnoid bleed, but excluding transient ischemic attacks [25] $C V A$ cerebrovascular accident, $C N S$ central nervous system, IC intermediate care, ICU intensive care unit, $P E$ pulmonary embolism, SSI surgical site infection, VTE venous thromboembolism
Hispanic White subjects, 36,970 (17.4\%) subjects were nonHispanic blacks, 28,673 (13.5\%) subjects were Hispanic, $985(0.5 \%)$ subjects were non-Hispanic Asians, and 1335 $(0.6 \%)$ subjects had other racial self-identification. The mean preoperative BMI closest to bariatric surgery was 45.6 (SD 8.1), with a mean postoperative BMI of 42.9 (SD 7.6). Complete demographic and comorbidity information are shown in Table 3. 
Table 2 MBSAQIP variables used in multivariable logistic regression analysis

\begin{tabular}{lll}
\hline Sex & On dialysis & Chronic steroids \\
\hline Age & Renal insufficiency & Albumin \\
Race & Diabetes & Venous stasis \\
GERD & Current smoker within a year & Vein thrombosis \\
History of MI & COPD history & First assistant training level \\
Hypertension & Oxygen dependent & History of PE \\
Hyperlipidemia & Sleep apnea & Surgical approach \\
\hline
\end{tabular}

\section{Procedure and Surgical Approach Information}

Procedure and outcome information are also included in Table 3. A total of $149,035(70.0 \%)$ subjects underwent a sleeve gastrectomy (CPT 43775). A total of 62,651 (29.4\%) subjects underwent a Roux-en-Y gastric bypass (61,353 subjects with a CPT code of 43,644, and 1298 subjects with a CPT code of 43,645). A total of $1284(0.6 \%)$ subjects underwent a biliopancreatic diversion with duodenal switch (CPT 43845). A total of 204,703 (96.1\%) subjects had a conventional laparoscopic surgical approach and 8267 (3.9\%) subjects had a laparoscopic-assisted surgical approach.

\section{Outcome Information: 30-Day Readmission and Clavien-Dindo Grade Classification}

A 30-day readmission and Clavien-Dindo Grade Classification information are included in Tables 4 and 5. A total of 9667 subjects $(4.5 \%)$ had at least one readmission within 30 days of operation. A total of 193,116 (90.7\%) subjects had none of the complications associated with the five Clavien-Dindo grade classifications. A total of $206(0.1 \%)$ subjects died within 30 days of the operation, resulting in a Grade V classification. A total of $1123(0.5 \%)$ subjects had their most severe complication correspond to a Grade IV classification; 6497 (3.1\%) subjects had their most severe complication correspond to a Grade III classification; 2775 (1.3\%) subjects had their most severe complication correspond to a Grade II classification; and 9253 (4.3\%) subjects had their most severe complication correspond to a Grade I classification.

\section{Association Between Race and 30-Day Readmission}

The results of the multivariable logistic regression model assessing the relationship between race and 30-day readmission are summarized in Table 5. For Black individuals in the analysis dataset, the odds of readmission within 30 days of surgery were $39 \%$ higher $(\mathrm{OR}=1.39 ; 95 \% \mathrm{CI}=1.32,1.46$; $p<0.0001)$ compared with White individuals after adjusting for covariates. The other racial groups in the analysis dataset (Asian, Hispanic, Other) did not have a statistically significant difference in odds of 30-day readmission compared with White individuals.

\section{Association Between Race and Clavien-Dindo Grade Classification}

The results of the multivariable multinomial logistic regression model assessing the relationship between race and Clavien-Dindo grade are summarized in Table 6. After adjusting for covariates, the odds of having a Grade I, III, IV, or V complication as the most severe complication, compared with having no complication at all, were $21 \%$ (OR = $1.21 ; 95 \% \mathrm{CI}=1.15,1.28 ; p<0.0001), 21 \%(\mathrm{OR}=1.21 ; 95 \%$ $\mathrm{CI}=1.13,1.29 ; p<0.0001), 22 \%(\mathrm{OR}=1.22 ; 95 \% \mathrm{CI}=1.04$, $1.42 ; p=0.01)$, and $43 \%(\mathrm{OR}=1.43 ; 95 \% \mathrm{CI}=1.01,2.03$; $p=0.04$ ) higher for Black individuals compared with White individuals respectively. Hispanic individuals have 59\% higher odds of a Grade 3 complication as the most severe complication compared with no complication $(\mathrm{OR}=1.59$; $95 \% \mathrm{CI}=1.49,1.70 ; p<0.0001)$ when compared with White individuals.

\section{Discussion}

This study of nearly 213,000 individuals found significant disparities in perioperative complication events based on race in individuals that had a primary laparoscopic bariatric operation. Racial disparities have been previously demonstrated and continue to hold important implications due to the growing emphasis on surgical quality improvement and a culture of safety. Disparities can contribute to rising healthcare costs as postoperative complications are a major factor in overall expenditures and resource utilization [38-40]. The patient's perspective and quality of life beyond financial considerations is of great importance and should not be overlooked. Although many minor surgical complications may not have lasting consequences, more severe complications can have significant ramifications contributing to patient disability, stress, and survival. While a certain level of postoperative complications are to be expected, acceptance of complications may vary depending on the previous health of the patient. For example, patients 
Table 3 Demographic, comorbidity, and readmission characteristics (by race)

\begin{tabular}{|c|c|c|c|c|c|c|c|}
\hline & $\begin{array}{l}\text { Asian } \\
(N=985)\end{array}$ & $\begin{array}{l}\text { Black } \\
(N=36,970)\end{array}$ & $\begin{array}{l}\text { Hispanic } \\
(N=28,673)\end{array}$ & $\begin{array}{l}\text { Other } \\
(N=1335)\end{array}$ & $\begin{array}{l}\text { White } \\
(N=145,007)\end{array}$ & $\begin{array}{l}\text { Total } \\
(N=212,970)\end{array}$ & $p$ value \\
\hline \multicolumn{8}{|l|}{ Procedure (CPT) } \\
\hline Gastric bypass (43644) & $285(28.9 \%)$ & $8719(23.6 \%)$ & $8196(28.6 \%)$ & $518(38.8 \%)$ & $43,635(30.1 \%)$ & $61,353(28.8 \%)$ & \\
\hline Gastric bypass (43645) & $2(0.2 \%)$ & $194(0.5 \%)$ & $410(1.4 \%)$ & $4(0.3 \%)$ & $688(0.5 \%)$ & $1298(0.6 \%)$ & \\
\hline $\begin{array}{l}\text { Sleeve gastrectomy } \\
\text { (43775) }\end{array}$ & $692(70.3 \%)$ & $27,885(75.4 \%)$ & $19,888(69.4 \%)$ & $798(59.8 \%)$ & $99,772(68.8 \%)$ & $149,035(70.0 \%)$ & \\
\hline Duodenal switch (43845) & $6(0.6 \%)$ & $172(0.5 \%)$ & $179(0.6 \%)$ & $15(1.1 \%)$ & $912(0.6 \%)$ & $1284(0.6 \%)$ & \\
\hline \multicolumn{8}{|c|}{ Surgical approach } \\
\hline Conventional laparoscopic & $962(97.7 \%)$ & $35,837(96.9 \%)$ & $27,602(96.3 \%)$ & $\begin{array}{l}1281 \\
(96.0 \%)\end{array}$ & $139,021(95.9 \%)$ & $204,703(96.1 \%)$ & \\
\hline Laparoscopic assisted & $23(2.3 \%)$ & $1133(3.1 \%)$ & $1071(3.7 \%)$ & $54(4.0 \%)$ & $5986(4.1 \%)$ & $8267(3.9 \%)$ & \\
\hline 30-day readmission & $38(3.9 \%)$ & $2223(6.0 \%)$ & $1198(4.2 \%)$ & $53(4.0 \%)$ & $6155(4.2 \%)$ & $9667(4.5 \%)$ & \\
\hline Sex (male) & $283(28.7 \%)$ & $5197(14.1 \%)$ & $5760(20.1 \%)$ & $289(21.6 \%)$ & $32,949(22.7 \%)$ & $44,478(20.9 \%)$ & \\
\hline Age, mean (SD) & $42.3(11.2)$ & $42.8(10.9)$ & $40.7(11.2)$ & $42.7(11.1)$ & $45.9(12.1)$ & $44.6(12.0)$ & \\
\hline Range & $(19.9-73.5)$ & $(18.0-79.6)$ & $(18.0-80.0)$ & $(18.0-76.3)$ & $(18.0-80.0)$ & $(18.0-80.0)$ & \\
\hline $\begin{array}{l}\text { BMI (preoperative), mean } \\
\text { (SD) }\end{array}$ & $43.4(7.9)$ & $47.3(8.8)$ & $44.9(7.8)$ & $47.0(9.2)$ & $45.3(8.0)$ & $45.6(8.1)$ & $<0.0001^{1}$ \\
\hline Range & $(30.0-98.5)$ & $(28.4-147.1)$ & $(27.0-105.5)$ & $(30.3-100.2)$ & $(27.5-121.2)$ & $(27.0-147.1)$ & \\
\hline $\begin{array}{l}\text { BMI (postoperative), mean } \\
\quad \text { (SD) }\end{array}$ & $40.5(7.2)$ & $44.5(8.2)$ & $42.1(7.2)$ & $44.3(8.7)$ & $42.6(7.4)$ & $42.9(7.6)$ & \\
\hline Range & $(24.2-95.8)$ & $(19.1-148.6)$ & $(19.3-95.9)$ & $(25.4-95.0)$ & $(18.5-123.7)$ & $(18.5-148.6)$ & \\
\hline GERD & $202(20.5 \%)$ & $9473(25.6 \%)$ & $7032(24.5 \%)$ & $356(26.7 \%)$ & $50,062(34.5 \%)$ & $67,125(31.5 \%)$ & \\
\hline History of MI & $8(0.8 \%)$ & $379(1.0 \%)$ & $208(0.7 \%)$ & $16(1.2 \%)$ & $2387(1.6 \%)$ & $2998(1.4 \%)$ & \\
\hline Hypertension & $462(46.9 \%)$ & $20,465(55.4 \%)$ & $11,180(39.0 \%)$ & $638(47.8 \%)$ & $73,287(50.5 \%)$ & $106,032(49.8 \%)$ & $<0.0001^{2}$ \\
\hline Hyperlipidemia & $282(28.6 \%)$ & $7378(20.0 \%)$ & $5313(18.5 \%)$ & $320(24.0 \%)$ & $39,963(27.6 \%)$ & $53,256(25.0 \%)$ & $<0.0001^{2}$ \\
\hline On dialysis & $5(0.5 \%)$ & $246(0.7 \%)$ & $82(0.3 \%)$ & $8(0.6 \%)$ & $229(0.2 \%)$ & $570(0.3 \%)$ & $<0.0001^{2}$ \\
\hline Renal insufficiency & $13(1.3 \%)$ & $420(1.1 \%)$ & $134(0.5 \%)$ & $15(1.1 \%)$ & $826(0.6 \%)$ & $1408(0.7 \%)$ & \\
\hline Diabetes & & & & & & & $<0.0001^{2}$ \\
\hline Insulin & $123(12.5 \%)$ & $3354(9.1 \%)$ & $2336(8.1 \%)$ & $159(11.9 \%)$ & $13,231(9.1 \%)$ & $19,203(9.0 \%)$ & \\
\hline Non-insulin & $253(25.7 \%)$ & $6655(18.0 \%)$ & $5360(18.7 \%)$ & $280(21.0 \%)$ & $26,016(17.9 \%)$ & $38,564(18.1 \%)$ & \\
\hline $\begin{array}{l}\text { Current smoker within } \\
1 \text { year }\end{array}$ & $94(9.5 \%)$ & $3032(8.2 \%)$ & $2326(8.1 \%)$ & $136(10.2 \%)$ & $13,762(9.5 \%)$ & $19,350(9.1 \%)$ & \\
\hline COPD history & $1(0.1 \%)$ & $596(1.6 \%)$ & $235(0.8 \%)$ & $23(1.7 \%)$ & $3048(2.1 \%)$ & $3903(1.8 \%)$ & $<0.0001^{2}$ \\
\hline Oxygen dependent & $0(0.0 \%)$ & $245(0.7 \%)$ & $118(0.4 \%)$ & $6(0.4 \%)$ & $1388(1.0 \%)$ & $1757(0.8 \%)$ & \\
\hline Sleep apnea & $386(39.2 \%)$ & $12,923(35.0 \%)$ & $8792(30.7 \%)$ & $547(41.0 \%)$ & $58,347(40.2 \%)$ & $80,995(38.0 \%)$ & \\
\hline $\begin{array}{l}\text { Steroid/immunosuppressant } \\
\text { use }\end{array}$ & $14(1.4 \%)$ & $672(1.8 \%)$ & $394(1.4 \%)$ & $32(2.4 \%)$ & $2467(1.7 \%)$ & $3579(1.7 \%)$ & $<0.0001^{2}$ \\
\hline $\begin{array}{l}\text { Albumin lab value, mean } \\
\text { (SD) }\end{array}$ & $4.1(0.4)$ & $3.9(0.4)$ & $4.1(0.4)$ & $4.0(0.4)$ & $4.1(0.4)$ & $4.0(0.4)$ & \\
\hline Range & $(2.2-5.2)$ & $(1.0-10.0)$ & $(1.0-9.4)$ & $(2.8-7.4)$ & $(1.0-10.0)$ & $(1.0-10.0)$ & \\
\hline Venous stasis & $13(1.3 \%)$ & $307(0.8 \%)$ & $283(1.0 \%)$ & $29(2.2 \%)$ & $2176(1.5 \%)$ & $2808(1.3 \%)$ & \\
\hline Vein thrombosis & $5(0.5 \%)$ & $638(1.7 \%)$ & $242(0.8 \%)$ & $12(0.9 \%)$ & $2767(1.9 \%)$ & $3664(1.7 \%)$ & \\
\hline History of PE & $3(0.3 \%)$ & $495(1.3 \%)$ & $138(0.5 \%)$ & $8(0.6 \%)$ & $1945(1.3 \%)$ & $2589(1.2 \%)$ & $<0.0001^{2}$ \\
\hline \multicolumn{8}{|l|}{ First assist training level } \\
\hline Attending - other & $45(4.6 \%)$ & $2172(5.9 \%)$ & $2785(9.7 \%)$ & $68(5.1 \%)$ & $7905(5.5 \%)$ & $12,975(6.1 \%)$ & \\
\hline $\begin{array}{l}\text { Attending - weight loss } \\
\text { surgeon }\end{array}$ & $185(18.8 \%)$ & $5604(15.2 \%)$ & $4249(14.8 \%)$ & $252(18.9 \%)$ & $24,932(17.2 \%)$ & $35,222(16.5 \%)$ & \\
\hline $\begin{array}{l}\text { Minimally invasive } \\
\text { surgery fellow }\end{array}$ & $106(10.8 \%)$ & $3522(9.5 \%)$ & $2953(10.3 \%)$ & $77(5.8 \%)$ & $11,959(8.2 \%)$ & $18,617(8.7 \%)$ & \\
\hline None (scrub tech/RN only) & $106(10.8 \%)$ & $5639(15.3 \%)$ & $3216(11.2 \%)$ & $210(15.7 \%)$ & $23,420(16.2 \%)$ & $32,591(15.3 \%)$ & \\
\hline $\begin{array}{l}\text { Physician assistant/nurse } \\
\text { practitioner/registered } \\
\text { nurse }\end{array}$ & $390(39.6 \%)$ & $12,666(34.3 \%)$ & $9032(31.5 \%)$ & $626(46.9 \%)$ & $57,636(39.7 \%)$ & $80,350(37.7 \%)$ & \\
\hline Resident (PGY 1-5+) & $153(15.5 \%)$ & $7367(19.9 \%)$ & $6438(22.5 \%)$ & $102(7.6 \%)$ & $19,155(13.2 \%)$ & $33,215(15.6 \%)$ & \\
\hline
\end{tabular}

$S D$ standard deviation, GERD gastroesophageal reflux disease, $H L D$ hyperlipidemia, $M I$ myocardial infarction, $C O P D$ chronic obstructive pulmonary disease, $P E$ pulmonary embolism, $P G Y$ postgraduate year

${ }^{1}$ Kruskal Wallis

${ }^{2}$ Chi-square

with dramatic improvement of their clinical condition after bariatric surgery may be more willing to accept minor complications as a valid trade-off for improved health as a result of the weight loss operation. 


\section{1,972 assessed for eligibility from 2015 and 2016 MBSAQIP datasets \\ 82,291 excluded \\ - Did not meet inclusion criteria of: \\ o primary laparoscopic Roux-en-Y gastric bypass, sleeve gastrectomy, or duodenal switch}

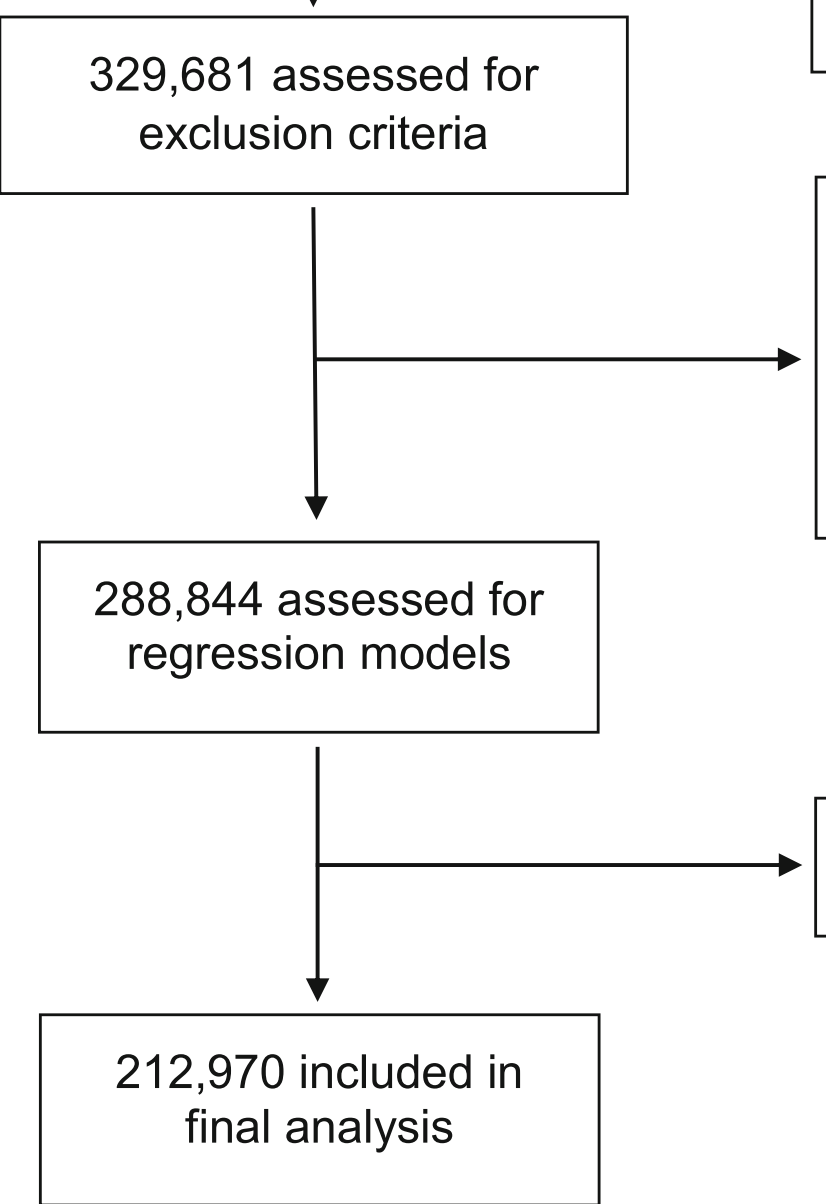

\section{5,874 excluded due to incomplete data}

Fig. 1 CONSORT flow diagram

Bariatric surgery remains safe with relatively low shortterm mortality rates ranging from 0.09 to $0.2 \%$ [8, 14-16, 41]. These figures are consistent with our findings from the MBSAQIP dataset. Published rates for complications are less clear and interpretations can be confusing as they often vary depending on loose definitions and the specific operations included in the study.

Previous studies have examined racial disparities specific to bariatric surgery outcomes. In 2013, using data extracted from the Nationwide Inpatient Sample between 1999 and 2007, Nguyen et al. [16] found that Black individuals had $73 \%$ higher odds of 30-day mortality and the findings were even more pronounced for males. Their analysis included over
82,291 excluded due to:

- revision/conversion

- $\mathrm{BMI}<18.5$

- age $<18$

- unknown race

\section{5,874 excluded due to incomplete data}

100,000 predominately restrictive procedures with some mixed/malabsorptive procedures. Both open and laparoscopic approaches were included. Sheka et al. [14], using MBSAQIP data from 2015, similarly found that Black patients demonstrated increased mortality after laparoscopic sleeve gastrectomy, but not for laparoscopic gastric bypass. They also found that Black patients had longer hospital lengths of stay, higher readmission rates, and higher reoperation rates after laparoscopic sleeve gastrectomy. Interestingly, Wood et al. [15] recently published a study that failed to show any difference in mortality or serious complications between Black and White patients after primary gastric bypass, sleeve gastrectomy, or adjustable gastric banding using data from the Michigan 
Table 4 Clavien-Dindo characteristics (by race)

\begin{tabular}{|c|c|c|c|c|c|c|}
\hline & $\begin{array}{l}\text { Asian } \\
(N=985)\end{array}$ & $\begin{array}{l}\text { Black } \\
(N=36,970)\end{array}$ & $\begin{array}{l}\text { Hispanic } \\
(N=28,673)\end{array}$ & $\begin{array}{l}\text { Other } \\
(N=1335)\end{array}$ & $\begin{array}{l}\text { White } \\
(N=145,007)\end{array}$ & $\begin{array}{l}\text { Total } \\
(N=212,970)\end{array}$ \\
\hline Clavien-Dindo Grade I & $33(3.4 \%)$ & $1984(5.4 \%)$ & $1232(4.3 \%)$ & $62(4.6 \%)$ & $5942(4.1 \%)$ & $9253(4.3 \%)$ \\
\hline Superficial incisional SSI & $9(0.9 \%)$ & $146(0.4 \%)$ & $139(0.5 \%)$ & $10(0.7 \%)$ & $966(0.7 \%)$ & $1270(0.6 \%)$ \\
\hline Dehydration treatment & $11(2.2 \%)$ & $1118(5.8 \%)$ & $570(3.8 \%)$ & $33(5.0 \%)$ & $3099(4.2 \%)$ & $4831(4.4 \%)$ \\
\hline Emergency department visit & $23(4.5 \%)$ & $1599(8.2 \%)$ & $1015(6.7 \%)$ & $56(8.5 \%)$ & $4559(6.1 \%)$ & $7252(6.6 \%)$ \\
\hline Peripheral nerve injury & $0(0.0 \%)$ & $1(0.0 \%)$ & $0(0.0 \%)$ & $0(0.0 \%)$ & $12(0.0 \%)$ & $13(0.0 \%)$ \\
\hline Clavien-Dindo Grade II & $13(1.3 \%)$ & $466(1.3 \%)$ & $334(1.2 \%)$ & $22(1.6 \%)$ & $1940(1.3 \%)$ & $2775(1.3 \%)$ \\
\hline Pneumonia & $6(0.6 \%)$ & $254(0.7 \%)$ & $214(0.7 \%)$ & $6(0.4 \%)$ & $1065(0.7 \%)$ & $1545(0.7 \%)$ \\
\hline Blood transfusion & $9(0.7 \%)$ & $356(0.7 \%)$ & $278(0.7 \%)$ & $13(0.7 \%)$ & $1461(0.7 \%)$ & $2117(0.7 \%)$ \\
\hline Urinary tract infection & $6(0.6 \%)$ & $138(0.4 \%)$ & $97(0.3 \%)$ & $7(0.5 \%)$ & $560(0.4 \%)$ & $808(0.4 \%)$ \\
\hline VTE requiring treatment & $1(0.1 \%)$ & $85(0.2 \%)$ & $67(0.2 \%)$ & $3(0.2 \%)$ & $315(0.2 \%)$ & $471(0.2 \%)$ \\
\hline Anticoagulation for VTE/PE & $3(0.3 \%)$ & $210(0.6 \%)$ & $134(0.5 \%)$ & $6(0.4 \%)$ & $659(0.5 \%)$ & $1012(0.5 \%)$ \\
\hline Clostridium difficile & $0(0.0 \%)$ & $25(0.1 \%)$ & $9(0.1 \%)$ & $2(0.3 \%)$ & $156(0.2 \%)$ & $192(0.2 \%)$ \\
\hline Clavien-Dindo Grade III & $22(2.2 \%)$ & $1248(3.4 \%)$ & $1213(4.2 \%)$ & $27(2.0 \%)$ & $3987(2.7 \%)$ & $6497(3.1 \%)$ \\
\hline Wound disruption & $1(0.1 \%)$ & $22(0.1 \%)$ & $27(0.1 \%)$ & $1(0.1 \%)$ & $82(0.1 \%)$ & $133(0.1 \%)$ \\
\hline Incisional hernia & $1(0.1 \%)$ & $29(0.1 \%)$ & $17(0.1 \%)$ & $1(0.1 \%)$ & $140(0.1 \%)$ & $188(0.1 \%)$ \\
\hline Deep incisional SSI & $0(0.0 \%)$ & $31(0.1 \%)$ & $19(0.1 \%)$ & $2(0.1 \%)$ & $135(0.1 \%)$ & $187(0.1 \%)$ \\
\hline Organ/space SSI & $2(0.2 \%)$ & $68(0.2 \%)$ & $68(0.2 \%)$ & $2(0.1 \%)$ & $417(0.3 \%)$ & $557(0.3 \%)$ \\
\hline $\begin{array}{l}\text { Drain placement after } \\
30 \text { days }\end{array}$ & $2(0.2 \%)$ & $60(0.2 \%)$ & $464(1.6 \%)$ & $1(0.1 \%)$ & $301(0.2 \%)$ & $828(0.4 \%)$ \\
\hline Reoperation within 30 days & $11(1.1 \%)$ & $595(1.6 \%)$ & $396(1.4 \%)$ & $16(1.2 \%)$ & $2122(1.5 \%)$ & $3140(1.5 \%)$ \\
\hline Intervention within 30 days & $14(1.4 \%)$ & $810(2.2 \%)$ & $469(1.6 \%)$ & $18(1.3 \%)$ & $2320(1.6 \%)$ & $3631(1.7 \%)$ \\
\hline Clavien-Dindo Grade IV & $3(0.3 \%)$ & $236(0.6 \%)$ & $100(0.3 \%)$ & $4(0.3 \%)$ & $780(0.5 \%)$ & $1123(0.5 \%)$ \\
\hline Acute renal failure & $2(0.2 \%)$ & $35(0.1 \%)$ & $13(0.0 \%)$ & $0(0.0 \%)$ & $112(0.1 \%)$ & $162(0.1 \%)$ \\
\hline $\begin{array}{l}\text { Progressive renal } \\
\text { insufficiency }\end{array}$ & $1(0.1 \%)$ & $55(0.1 \%)$ & $15(0.1 \%)$ & $2(0.1 \%)$ & $104(0.1 \%)$ & $177(0.1 \%)$ \\
\hline Myocardial infarction & $2(0.2 \%)$ & $8(0.0 \%)$ & $8(0.0 \%)$ & $1(0.1 \%)$ & $70(0.0 \%)$ & $89(0.0 \%)$ \\
\hline Stroke/CVA & $0(0.0 \%)$ & $5(0.0 \%)$ & $1(0.0 \%)$ & $0(0.0 \%)$ & $19(0.0 \%)$ & $25(0.0 \%)$ \\
\hline Ventilator $>48 \mathrm{~h}$ & $0(0.0 \%)$ & $2(0.0 \%)$ & $0(0.0 \%)$ & $0(0.0 \%)$ & $1(0.0 \%)$ & $3(0.0 \%)$ \\
\hline Unplanned intubation & $1(0.1 \%)$ & $77(0.2 \%)$ & $24(0.1 \%)$ & $2(0.1 \%)$ & $284(0.2 \%)$ & $388(0.2 \%)$ \\
\hline Pulmonary embolism & $1(0.1 \%)$ & $67(0.2 \%)$ & $25(0.1 \%)$ & $1(0.1 \%)$ & $157(0.1 \%)$ & $251(0.1 \%)$ \\
\hline Cardiac arrest & $0(0.0 \%)$ & $15(0.0 \%)$ & $7(0.0 \%)$ & $1(0.1 \%)$ & $52(0.0 \%)$ & $75(0.0 \%)$ \\
\hline Coma $>24 \mathrm{~h}$ & $0(0.0 \%)$ & $4(0.0 \%)$ & $1(0.0 \%)$ & $0(0.0 \%)$ & $9(0.0 \%)$ & $14(0.0 \%)$ \\
\hline Sepsis & $0(0.0 \%)$ & $39(0.1 \%)$ & $31(0.1 \%)$ & $4(0.3 \%)$ & $192(0.1 \%)$ & $266(0.1 \%)$ \\
\hline Septic shock & $0(0.0 \%)$ & $40(0.1 \%)$ & $31(0.1 \%)$ & $4(0.3 \%)$ & $198(0.1 \%)$ & $273(0.1 \%)$ \\
\hline Clavien-Dindo Grade V & $2(0.2 \%)$ & $46(0.1 \%)$ & $13(0.0 \%)$ & $2(0.1 \%)$ & $143(0.1 \%)$ & $206(0.1 \%)$ \\
\hline 30-day mortality & $2(0.2 \%)$ & $46(0.1 \%)$ & $13(0.0 \%)$ & $2(0.1 \%)$ & $143(0.1 \%)$ & $206(0.1 \%)$ \\
\hline
\end{tabular}

Bariatric Surgery Collaborative. There was a small increase in rates of any complication and reoperation, but no difference in serious complications after adjusting for surgeon and hospital effects. Readmission rates were significantly higher for Black patients after adjusting for patient, surgeon, and hospital factors.

Reporting surgical complications can be confusing and controversial as definitions are wide and varied, making comparisons difficult. The American Society for Metabolic and Bariatric Surgery has established recommendations for a standardized framework for reporting early complications (within
30 days of surgery) using "major" and "minor" designations [42]. Major complications are those that result in a prolonged hospital stay (longer than 7 days), anticoagulation, reoperation, or reintervention; all other complications are classified as minor. Despite these recommendations, the lack of granularity in the "major/minor" system, and variations in results found in the literature make it difficult to attain accurate assessments and draw conclusions.

The Clavien-Dindo grade classification, originally developed in 1992 and revised in 2004, establishes a validated grading system with well-defined definitions for perioperative 
Table 5 Relationship between race and 30-day readmission

\begin{tabular}{|c|c|c|}
\hline Covariates & Adjusted OR (95\% CI) & $P$ value \\
\hline $\begin{array}{l}\text { Race* } \\
\text { White }\end{array}$ & Reference & \\
\hline Asian & $0.95(0.68,1.31)$ & 0.74 \\
\hline Black & $1.39(1.32,1.46)$ & $<0.0001$ \\
\hline Hispanic & $1.02(0.96,1.09)$ & 0.50 \\
\hline Other & $0.93(0.70,1.22)$ & 0.59 \\
\hline Age of patient at time of surgery (per 1-year increase) & $0.99(0.99,0.99)$ & $<0.0001$ \\
\hline $\begin{array}{l}\text { Sex } \\
\text { Female }\end{array}$ & Reference & \\
\hline Male & $0.85(0.81,0.90)$ & $<0.0001$ \\
\hline BMI (per 1-year increase) & $1.01(1.00,1.01)$ & $<0.0001$ \\
\hline GERD (reference: no history in last 30 days) & $1.43(1.37,1.50)$ & $<0.0001$ \\
\hline History of MI (reference: no history) & $1.26(1.09,1.46)$ & 0.002 \\
\hline Hypertension requiring medication (reference: none at time of operation) & $1.08(1.03,1.13)$ & 0.003 \\
\hline Hyperlipidemia (reference: none at time of operation) & $1.08(1.02,1.14)$ & 0.008 \\
\hline Dialysis (reference: none at time of operation) & $1.53(1.15,2.03)$ & 0.004 \\
\hline Renal insufficiency (reference: none at time of operation) & $1.49(1.23,1.81)$ & $<0.0001$ \\
\hline $\begin{array}{l}\text { Diabetes* } \\
\text { No }\end{array}$ & Reference & \\
\hline Non-Insulin & $1.07(1.01,1.13)$ & 0.02 \\
\hline Insulin & $1.50(1.40,1.61)$ & $<0.0001$ \\
\hline Current smoker within 1 year (reference: non-smoker within 1 year) & $1.06(0.98,1.13)$ & 0.13 \\
\hline History of COPD (reference: no history) & $1.27(1.12,1.45)$ & 0.0003 \\
\hline Oxygen dependent (reference: none at time of operation) & $1.48(1.24,1.76)$ & $<0.0001$ \\
\hline Sleep apnea (reference: none at time of operation) & $1.08(1.03,1.13)$ & 0.0009 \\
\hline Steroid/immunosuppressant use (reference: none at time of operation) & $1.16(1.01,1.34)$ & 0.03 \\
\hline Albumin lab value (per one unit increase) & $0.81(0.77,0.85)$ & $<0.0001$ \\
\hline Venous stasis (reference: none at time of operation) & $1.06(0.91,1.25)$ & 0.45 \\
\hline Vein thrombosis (reference: none at time of operation) & $1.75(1.54,1.98)$ & $<0.0001$ \\
\hline History of PE (reference: no history) & $1.40(1.21,1.63)$ & $<0.0001$ \\
\hline $\begin{array}{l}\text { First assistant training level* } \\
\text { None (no assist or scrub tech/RN only) }\end{array}$ & Reference & \\
\hline Other & $0.91(0.82,1.01)$ & 0.07 \\
\hline Resident (PGY 1-5+) & $1.14(1.06,1.23)$ & 0.0005 \\
\hline Physician assistant/nurse practitioner/registered nurse & $1.05(0.98,1.12)$ & 0.16 \\
\hline Minimally invasive surgery fellow & $1.18(1.08,1.28)$ & 0.0002 \\
\hline Weight loss surgeon & $1.08(1.01,1.17)$ & 0.03 \\
\hline $\begin{array}{l}\text { Surgical approach } \\
\text { Conventional laparoscopic (thoracoscopic) }\end{array}$ & Reference & \\
\hline Laparoscopic assisted (thoracoscopic assisted) & $0.96(0.86,1.07)$ & 0.43 \\
\hline
\end{tabular}

Odds ratios and $95 \%$ confidence intervals from modeling respective non-White racial statuses compared with White racial status on 30-day readmission after adjusting for covariates $(N=212,970)$. (*Denotes a $p<0.0001$ for type 3 analysis of effects for variables with multiple categorical levels)

complications [25]. According to this system, a complication is defined as any deviation from the postoperative course. This classification focuses primarily on therapeutic consequences (e.g., intensive care transfers) or surgical interventions, as these events are associated with increased mortality, patient stress, and resource consumption [25]. In addition, this system allows for more detailed, reproducible, and comparable stratification of complications across centers and disciplines.

Our findings are consistent with those reported by others regarding readmission rates among Black patients compared with White patients, with 39\% higher odds of readmission after adjusting for covariates. Readmission 
Table 6 Odds ratios of ClavienDindo complications by race

Odds ratios and $95 \%$ confidence intervals from modeling respective non-White racial statuses compared with White racial status on Clavien-Dindo grading (compared with "No Grade") after adjusting for covariates $(N=212,970)$

\begin{tabular}{llll}
\hline Covariate & OR $(95 \%$ CI $)$ & $P$ value & Bonferroni \\
\hline Clavien-Dindo Grade 1: (Asian vs White) & $0.83(0.58,1.17)$ & 0.28 & $\mathrm{NS}$ \\
Clavien-Dindo Grade 2: (Asian vs White) & $1.06(0.61,1.83)$ & 0.85 & $\mathrm{NS}$ \\
Clavien-Dindo Grade 3: (Asian vs White) & $0.86(0.56,1.31)$ & 0.48 & $\mathrm{NS}$ \\
Clavien-Dindo Grade 4: (Asian vs White) & $0.62(0.20,1.93)$ & 0.41 & $\mathrm{NS}$ \\
Clavien-Dindo Grade 5: (Asian vs White) & $2.77(0.68,11.35)$ & 0.16 & $\mathrm{NS}$ \\
Clavien-Dindo Grade 1: (Black vs White) & $1.21(1.15,1.28)$ & $<0.0001$ & $\mathrm{~S}$ \\
Clavien-Dindo Grade 2: (Black vs White) & $0.94(0.85,1.05)$ & 0.28 & $\mathrm{NS}$ \\
Clavien-Dindo Grade 3: (Black vs White) & $1.21(1.13,1.29)$ & $<0.0001$ & $\mathrm{~S}$ \\
Clavien-Dindo Grade 4: (Black vs White) & $1.22(1.04,1.42)$ & 0.01 & $\mathrm{NS}$ \\
Clavien-Dindo Grade 5: (Black vs White) & $1.43(1.01,2.03)$ & 0.04 & $\mathrm{NS}$ \\
Clavien-Dindo Grade 1: (Hispanic vs White) & $1.04(0.97,1.11)$ & 0.28 & $\mathrm{NS}$ \\
Clavien-Dindo Grade 2: (Hispanic vs White) & $0.95(0.84,1.07)$ & 0.40 & $\mathrm{NS}$ \\
Clavien-Dindo Grade 3: (Hispanic vs White) & $1.59(1.49,1.70)$ & $<0.0001$ & $\mathrm{~S}$ \\
Clavien-Dindo Grade 4: (Hispanic vs White) & $0.87(0.70,1.07)$ & 0.19 & $\mathrm{NS}$ \\
Clavien-Dindo Grade 5: (Hispanic vs White) & $0.74(0.42,1.32)$ & 0.31 & $\mathrm{NS}$ \\
Clavien-Dindo Grade 1: (Other vs White) & $1.08(0.84,1.40)$ & 0.55 & $\mathrm{NS}$ \\
Clavien-Dindo Grade 2: (Other vs White) & $1.28(0.84,1.95)$ & 0.26 & $\mathrm{NS}$ \\
Clavien-Dindo Grade 3: (Other vs White) & $0.73(0.50,1.07)$ & 0.11 & $\mathrm{NS}$ \\
Clavien-Dindo Grade 4: (Other vs White) & $0.60(0.22,1.60)$ & 0.30 & $\mathrm{NS}$ \\
Clavien-Dindo Grade 5: (Other vs White) & $1.70(0.42,6.91)$ & 0.46 & $\mathrm{NS}$ \\
\hline
\end{tabular}

Multivariable multinomial logistic regression model assessing the relationship between race and Clavien-Dindo grade after adjusting for the following covariates: sex, age, race, GERD, history of MI, hypertension, hyperlipidemia, on dialysis, renal insufficiency, diabetes, current smoker within 1 year, COPD history, oxygen dependent, sleep apnea, chronic steroids, albumin, venous stasis, vein thrombosis, history of PE, first assistant training level, and surgical approach

$N S$ not significant, $S$ significant

stands a significant contributor to resource utilization and cause of distress to the patient. Previous studies have demonstrated that resource utilization is in part affected by racial differences in socioeconomic factors such as social support, access to transportation, payor status, and primary care physician access [43-45]. Racial differences between the patient and surgeon can lead to communication barriers and poor understanding of postoperative instructions. Such breakdowns have been shown to contribute to higher readmission rates in Black patients [46] and may also be present in the bariatric population. Geographic, socioeconomic, or payor status information is not included in the MBSAQIP registry. Information regarding the potential reason for readmission including whether the readmission was likely related to surgery, days from operation to readmission, or most likely reason for readmission are available in the supplemental file; however, these variables were not included in our analysis. Further investigation may elucidate possible causes and potential interventions.
The more alarming results of this study are the disparities in complications for Black patients. Multinomial logistic regression models showed that Black individuals had higher odds of Clavien-Dindo Grade I, III, IV, and V as their most severe complication compared with White individuals. Specifically, compared with having no complications, Black subjects had $43 \%$ higher odds of 30-day mortality compared with White patients (Clavien-Dindo Grade V). The mixed results from previous studies may be due to low complication rates, resulting in a lack of power to detect statistically significant differences. While previous studies often categorized complications as minor or major, we believe that grouping individual complication events into Clavien-Dindo grades allows for more precise categorizations.

The finding that Hispanic patients have 59\% higher odds of a Grade III complication is intriguing. By definition, Grade III complications are complications that require surgical, endoscopic, or radiologic intervention, likely representing leaks or bleeding. Further investigation into the individual components 
included in this grade demonstrated that the bulk of the occurrences were related to having a drain in place 30 days after surgery. Specific reasons behind this are unclear and may reflect an increased incidence of postoperative leak. The MBSAQIP does not have a specific variable for leaks and many authors use drain in place after 30 days as a surrogate $[47,48]$. However, this is at odds with the finding that Hispanic patients have less organ space infections; one would also expect to see an increase in Hispanic patients with a leak.

There are several limitations of this study. While the MBSAQIP data set is the most comprehensive bariatric database available, it is devoid of information that may identify surgeon training level and experience [12, 49-51]. Details of patient income level, insurance status, and geographic location are also absent from the dataset, and may provide additional information regarding socioeconomic factors. While the proportions found in the data set may approximate the general population as a whole [52], estimates indicate that obesity is much higher in Blacks and Hispanics [53] suggesting a disparity in access. While only 5 of the 20 comparisons garnered statistically significant results (Table 6), three remained (Grades I, III, and IV) remained significant for Black patients after adjusting for multiple comparisons using the Bonferroni approach, suggesting increased risk of a complication when separated by severity. The considerably smaller sizes of the Asian and Other race groups likely negatively influenced our ability to make any meaningful conclusions compared with the White and Black groups. Of note, we feel that the study is adequately powered regarding the comparison of White and Black patients and these demographics comprise the majority of bariatric operations in the USA.

Significant challenges exist in surgical health disparities research and intervention. Most studies, including this one, utilize retrospective data often collected for other purposes that lack important information on race. A meta-analysis by Haider et al. [12] systematically describes several factors that contribute to disparities including insurance and socioeconomic status, patient willingness to undergo surgery, advanced presentation and disease burden, access to and type of surgery selected, surgeon volume and experience, and hospital volume. Some disparities are known to persist even after controlling for insurance status and socioeconomic status [54, 55]. These conflicting findings highlight the complexity of the topic and prospective studies are needed to better understand patient and surgeon decision making, develop standardized management plans, and to assess the impact of interventions. Enhanced recovery pathways have demonstrated significant improvements in perioperative care by standardizing care [56] and adoption of standardized protocols may help reduce underlying implicit bias that can result in outcome disparities $[57,58]$.

Improved communication decreases health disparities and further promotes equality $[59,60]$. The ACCURE Trial was able to reduce the racial disparities in breast and lung cancer by implementing a multifaceted, system-based protocol with race-specific alerts for improved communication between surgeon, patient, and other team members [61]. Similar principles may be useful for bariatric patients.

Reducing inequity in surgery access has been shown to eliminate disparities and could stand as a possible target of intervention as racial minorities undergo bariatric surgery at disproportionately low rates [62]. Further information regarding surgeon and hospital volume may be useful in identifying and improving structural mechanisms that limit access to minority populations. In an analysis of coronary bypass patients, Kim et al. [62] demonstrated a significant relationship between hospital volume and mortality after controlling for patient factors as the benefit of higher-volume hospitals was stronger for Blacks than Whites. One limitation of the MBSAQIP is its lack of surgeon and hospital information regarding volume, region, and patient socioeconomic specifics which may be used to identify possible solutions.

This study utilizes a large national registry with robust and validated data and adds to a growing body of literature demonstrating racial disparities involving bariatric surgery. Future research should work to provide qualitative information to provide standardized care and improved communication.

\section{Conclusion}

Bariatric surgery remains effective and safe with relatively low complication rates. However, significant racial disparities exist. Compared with White individuals, Black individuals have higher odds of readmission and many Clavien-Dindo grades, including higher odds of death compared with White patients. Compared with White patients, Hispanic patients have higher odds of moderately severe complications requiring invasive intervention. Specific causes or contributory factors of these disparities are beyond the limitations of the dataset and stands as a topic for future inquiry.

Funding Information The Duke BERD Methods Core's support of this project was made possible (in part) by Grant Number UL1TR002553 from the National Center for Advancing Translational Sciences (NCATS) of the National Institutes of Health (NIH) and NIH Roadmap for Medical Research.

\section{Compliance with Ethical Standards}

Conflict of Interest Dana Portenier has received research support from Medtronic, and education grant from Levita and Gore. Has received honorarium as a speaker for Nova Nordisk, and as a consultant for Medtronic and Intuitive. Alfredo D. Guerron has received honorarium as a consultant for Levita and as a speaker for Gore and Medtronic. The other authors declare that they have no conflict of interest. 
Ethical Approval For this retrospective study, formal consent is not required.

Informed Consent Informed consent does not apply.

Disclaimer Its contents are solely the responsibility of the authors and do not necessarily represent the official views of NCATS or NIH.

\section{References}

1. Hales CM, Carroll MD, Fryar CD, et al. Prevalence of obesity among adults and Youth: United States, 2015-2016. NCHS Data Brief No.288. 2017;1-8.

2. Currie A, Chetwood A, Ahmed AR. Bariatric surgery and renal function. Obes Surg. 2011;21(4):528-39.

3. Nostedt JJ, Switzer NJ, Gill RS, et al. The effect of bariatric surgery on the spectrum of fatty liver disease. Can J Gastroenterol Hepatol. 2016;2016:2059245.

4. Sarkhosh K, Switzer NJ, El-Hadi M, et al. The impact of bariatric surgery on obstructive sleep apnea: a systematic review. Obes Surg. 2013;23(3):414-23.

5. Tan O, Carr BR. The impact of bariatric surgery on obesity-related infertility and in vitro fertilization outcomes. Semin Reprod Med. 2012;30(6):517-28

6. Yska JP, van Roon EN, de Boer A, et al. Remission of type 2 diabetes mellitus in patients after different types of bariatric surgery: a population-based cohort study in the United Kingdom. JAMA Surg. 2015;150(12):1126-33.

7. Guerron AD, Ortega CB, Lee HJ, et al. Asthma medication usage is significantly reduced following bariatric surgery. Surg Endosc. 2019;33(6):1967-75.

8. Longitudinal Assessment of Bariatric Surgery, C, Flum DR, Belle $\mathrm{SH}$, et al. Perioperative safety in the longitudinal assessment of bariatric surgery. N Engl J Med. 2009;361(5):445-54.

9. Nguyen NT, Masoomi H, Magno CP, et al. Trends in use of bariatric surgery, 2003-2008. J Am Coll Surg. 2011;213(2):261-6.

10. Ravi P, Sood A, Schmid M, et al. Racial/ethnic disparities in perioperative outcomes of major procedures: results from the National Surgical Quality Improvement Program. Ann Surg. 2015;262(6): 955-64.

11. Sukumar S, Ravi P, Sood A, et al. Racial disparities in operative outcomes after major cancer surgery in the United States. World J Surg. 2015;39(3):634-43.

12. Haider AH, Scott VK, Rehman KA, et al. Racial disparities in surgical care and outcomes in the United States: a comprehensive review of patient, provider, and systemic factors. J Am Coll Surg. 2013;216(3):482-92. e12

13. Carden A, Blum K, Arbaugh CJ, et al. Low socioeconomic status is associated with lower weight-loss outcomes 10-years after Rouxen-Y gastric bypass. Surg Endosc. 2019;33(2):454-9.

14. Sheka AC, Kizy S, Wirth K, et al. Racial disparities in perioperative outcomes after bariatric surgery. Surg Obes Relat Dis. 2019;15(5): 786-93.

15. Wood MH, Carlin AM, Ghaferi AA, et al. Association of race with bariatric surgery outcomes. JAMA Surg. 2019;154(5):e190029.

16. Nguyen GC, Patel AM. Racial disparities in mortality in patients undergoing bariatric surgery in the U.S.A. Obes Surg. 2013;23(10): 1508-14.

17. Byrd AS, Toth AT, Stanford FC. Racial disparities in obesity treatment. Curr Obes Rep. 2018;7(2):130-8.

18. Worni M, Guller U, Maciejewski ML, et al. Racial differences among patients undergoing laparoscopic gastric bypass surgery: a population-based trend analysis from 2002 to 2008. Obes Surg. 2013;23(2):226-33.

19. Khorgami Z, Arheart KL, Zhang C, et al. Effect of ethnicity on weight loss after bariatric surgery. Obes Surg. 2015;25(5):769-76.

20. Coleman KJ, Huang YC, Hendee F, et al. Three-year weight outcomes from a bariatric surgery registry in a large integrated healthcare system. Surg Obes Relat Dis. 2014;10(3):396-403.

21. Istfan N, Anderson WA, Apovian C, et al. Racial differences in weight loss, hemoglobin $\mathrm{A} 1 \mathrm{C}$, and blood lipid profiles after Roux-en-Y gastric bypass surgery. Surg Obes Relat Dis. 2016;12(7):1329-36.

22. Turner PL, Oyetunji TA, Gantt G, et al. Demographically associated variations in outcomes after bariatric surgery. Am J Surg. 2011;201(4):475-80.

23. Sudan R, Winegar D, Thomas S, et al. Influence of ethnicity on the efficacy and utilization of bariatric surgery in the USA. J Gastrointest Surg. 2014;18(1):130-6.

24. Tiwari MM, Goede MR, Reynoso JF, et al. Differences in outcomes of laparoscopic gastric bypass. Surg Obes Relat Dis. 2011;7(3): 277-82.

25. Dindo D, Demartines N, Clavien PA. Classification of surgical complications: a new proposal with evaluation in a cohort of 6336 patients and results of a survey. Ann Surg. 2004;240(2): 205-13.

26. Miller-Matero LR, Tobin ET, Clark S, et al. Pursuing bariatric surgery in an urban area: gender and racial disparities and risk for psychiatric symptoms. Obes Res Clin Pract. 2016;10(1):56-62.

27. Craven MR, Kia L, O'Dwyer LC, et al. Systematic review: methodological flaws in racial/ethnic reporting for gastroesophageal reflux disease. Dis Esophagus. 2018;31(3). https://doi.org/10.1093/ dote/dox 154

28. Zhang ZM, Rautaharju PM, Prineas RJ, et al. Race and sex differences in the incidence and prognostic significance of Silent Myocardial Infarction in the Atherosclerosis Risk in Communities (ARIC) Study. Circulation. 2016;133(22):2141-8.

29. Fryar CD, Ostchega Y, Hales CM, et al. Hypertension prevalence and control among adults: United States, 2015-2016. NCHS Data Brief No.289. 2017;1-8.

30. Safford MM, Gamboa CM, Durant RW, et al. Race-sex differences in the management of hyperlipidemia: the reasons for Geographic and Racial Differences in Stroke study. Am J Prev Med. 2015;48(5):520-7.

31. Streja E, Nicholas SB, Norris KC. Controversies in timing of dialysis initiation and the role of race and demographics. Semin Dial. 2013;26(6):658-66.

32. Denker M, Boyle S, Anderson AH, et al. Chronic Renal Insufficiency Cohort Study (CRIC): overview and summary of selected findings. Clin J Am Soc Nephrol. 2015;10(11):2073-83.

33. CDC. National Diabetes Statistic Report. 2020 [cited 2020; Available from: https://www.cdc.gov/diabetes/data/statistics/ statistics-report.html.

34. Karriker-Jaffe KJ, Liu H, Johnson RM. Racial/ethnic differences in associations between neighborhood socioeconomic status, distress, and smoking among U.S. adults. J Ethn Subst Abus. 2016;15(1): 73-91.

35. Mamary AJ, Stewart JI, Kinney GL, et al. Race and gender disparities are evident in COPD underdiagnoses across all severities of measured airflow obstruction. Chronic Obstr Pulm Dis. 2018;5(3): 177-84.

36. Dudley KA, Patel SR. Disparities and genetic risk factors in obstructive sleep apnea. Sleep Med. 2016;18:96-102.

37. White RH, Keenan CR. Effects of race and ethnicity on the incidence of venous thromboembolism. Thromb Res. 2009;123(Suppl 4):S11-7. 
38. Lang M, Niskanen M, Miettinen P, et al. Outcome and resource utilization in gastroenterological surgery. Br J Surg. 2001;88(7): 1006-14.

39. Ferguson Jr TB, Ferguson CL, Crites K, et al. The additional hospital costs generated in the management of complications of pacemaker and defibrillator implantations. J Thorac Cardiovasc Surg. 1996;111(4):742-51. discussion 751-2

40. Mason LB, Garcia AG. Hospital costs of surgical complications. Arch Surg. 1984;119(9):1065-6.

41. Cardoso L, Rodrigues D, Gomes L, et al. Short- and long-term mortality after bariatric surgery: a systematic review and meta-analysis. Diabetes Obes Metab. 2017;19(9):1223-32.

42. Brethauer SA, Kim J, el Chaar M, et al. Standardized outcomes reporting in metabolic and bariatric surgery. Surg Obes Relat Dis. 2015;11(3):489-506.

43. Arbaje AI, Wolff JL, Yu Q, et al. Postdischarge environmental and socioeconomic factors and the likelihood of early hospital readmission among community-dwelling Medicare beneficiaries. Gerontologist. 2008;48(4):495-504.

44. Joynt KE, Jha AK. A path forward on Medicare readmissions. N Engl J Med. 2013;368(13):1175-7.

45. Shi L, Lebrun-Harris LA, Daly CA, et al. Reducing disparities in access to primary care and patient satisfaction with care: the role of health centers. J Health Care Poor Underserved. 2013;24(1):56-66.

46. Wong MS, Gudzune KA, Bleich SN. Provider communication quality: influence of patients' weight and race. Patient Educ Couns. 2015;98(4):492-8.

47. Mocanu V, Dang J, Ladak F, et al. Predictors and outcomes of leak after Roux-en-Y gastric bypass: an analysis of the MBSAQIP data registry. Surg Obes Relat Dis. 2019;15(3):396-403.

48. Alizadeh RF, Li S, Inaba C, et al. Risk factors for gastrointestinal leak after bariatric surgery: MBASQIP analysis. J Am Coll Surg. 2018;227(1):135-41.

49. Maruthappu M, Gilbert BJ, El-Harasis MA, et al. The influence of volume and experience on individual surgical performance: a systematic review. Ann Surg. 2015;261(4):642-7.

50. Goldberg I, Yang J, Park J, et al. Surgical trainee impact on bariatric surgery safety. Surg Endosc. 2019;33(9):3014-25.

51. Ilczyszyn A, Lynn W, Rasheed S, et al. Bariatric fellowship positively influences early outcomes for laparoscopic Roux-en-Y gastric bypass surgery over seven years of independent practice. Ann R Coll Surg Engl. 2018:100(8):1-5.

52. U.S. Census Bureau QuickFacts: United States. [cited 2018 July 1]; Available from: https://www.census.gov/quickfacts/fact/table/US/ PST045218.

53. Hales CM, Fryar CD, Carroll MD, et al. Differences in obesity prevalence by demographic characteristics and urbanization level among adults in the United States, 2013-2016. JAMA. 2018;319(23):2419-29.

54. Nathan H, Frederick W, Choti MA, et al. Racial disparity in surgical mortality after major hepatectomy. J Am Coll Surg. 2008;207(3): 312-9.

55. Bowman K, Telem DA, Hernandez-Rosa J, et al. Impact of race and socioeconomic status on presentation and management of ventral hernias. Arch Surg. 2010;145(8):776-80.

56. Malczak P, Pisarska M, Piotr M, et al. Enhanced recovery after bariatric surgery: systematic review and meta-analysis. Obes Surg. 2017;27(1):226-35.

57. Baptiste C, D'Alton ME. Applying patient safety to reduce maternal mortality. Obstet Gynecol Clin N Am. 2019;46(2):353-65.

58. Cronin KA, Howlader N, Stevens JL, et al. Racial disparities in the receipt of guideline care and cancer deaths for women with ovarian cancer. Cancer Epidemiol Biomark Prev. 2019;28(3):539-45.

59. Perez-Stable EJ, El-Toukhy S. Communicating with diverse patients: how patient and clinician factors affect disparities. Patient Educ Couns. 2018;101(12):2186-94.

60. Nathan AG, Marshall IM, Cooper JM, et al. Use of decision aids with minority patients: a systematic review. J Gen Intern Med. 2016;31(6):663-76.

61. Cykert S, Eng E, Manning MA, et al. A multi-faceted Intervention aimed at Black-White disparities in the treatment of early stage cancers: The ACCURE Pragmatic Quality Improvement trial. J Natl Med Assoc. 2019. https://doi.org/10.1016/j.jnma.2019.03.001

62. Hofmann LJ, Lee S, Waddell B, et al. Effect of race on colon cancer treatment and outcomes in the Department of Defense healthcare system. Dis Colon Rectum. 2010;53(1):9-15.

Publisher's Note Springer Nature remains neutral with regard to jurisdictional claims in published maps and institutional affiliations. 